\title{
MLPNN and kNN Based Classification of sEMG Signal for Myoelectric Control of Upper Limb Prosthesis
}

\author{
Sachin Negi \\ Dept. of Electrical Engineering \\ G. B. Pant Engineering College \\ Pauri- Garhwal, Uttarakhand, India \\ sachinnegi.coep@gmail.com
}

\author{
Yatindra Kumar \\ Dept. of Electrical Engineering \\ G. B. Pant Engineering College \\ Pauri- Garhwal, Uttarakhand, India \\ kyatindra@gmail.com
}

\author{
V. M. Mishra \\ Dept. of Electrical Engineering \\ G. B. Pant Engineering College \\ Pauri- Garhwal, Uttarakhand, India \\ vmm66@rediffmail.com
}

\begin{abstract}
Analysis of sEMG signal has been an emerging field for the myoelectric control of upper limb prosthesis. The objective of present work is to obtain the performance measures like accuracy, sensitivity, specificity and positive predictivity using MLPNN with back propagation algorithm. Using MLPNN classifier, an average classification accuracy of $\mathbf{9 3 . 7 1 \%}$ was achieved over ten subjects for the combination of [MAV1, WL, AAC, ZC, and WAMP] features. Next the classification accuracy is obtained with $\mathrm{kNN}$ classifier for $\mathrm{k}=3$, 5 , and 7 . The results showed that average classification accuracy of $93.06 \%$ is achieved using $\mathrm{kNN}$ and it is better than MLPNN in terms of time and simplicity.
\end{abstract}

Index Terms—sEMG; MLPNN; kNN; upper limb prosthesis.

\section{ABBREVIATIONS}

$\begin{array}{ll}\text { AAC } & \text { Average Amplitude Change } \\ \text { ANN } & \text { Artificial Neural Network } \\ \text { AR } & \text { Auto-Regressive } \\ \text { IAV } & \text { Integral of Absolute Value } \\ \text { kNN } & \text { k- Nearest Neighbors } \\ \text { LDA } & \text { Linear Discriminant Analysis } \\ \text { MAV } & \text { Mean Absolute Value } \\ \text { MAVS } & \text { Mean Absolute Value Slope } \\ \text { MES } & \text { Myoelectric signals } \\ \text { MLPNN } & \text { Multilayer Perceptron Neural Network } \\ \text { PCA } & \text { Principal Component Analysis } \\ \text { RMS } & \text { Root Mean Square } \\ \text { sEMG } & \text { Surface Electromyogram } \\ \text { SD } & \text { Standard Deviation } \\ \text { SSC } & \text { Slope Sign Changes } \\ \text { ULDA } & \text { Uncorrelated Linear } \\ \text { nalysis } & \\ \text { VAR } & \text { Variance } \\ \text { WAMP } & \text { Willison Amplitude } \\ \text { WL } & \text { Waveform Length } \\ \text { ZC } & \text { Zero Crossings }\end{array}$

\section{INTRODUCTION}

Prosthetic devices play a vital role in rehabilitation for the amputees who have lost their upper limbs due to several reasons. Nowadays, sEMG is the most dominant source of control signal to develop myoelectric upper limb prostheses. The success of myoelectric control for upper limb prostheses greatly depends on the effective feature extraction and the classification methods to achieve classification accuracy.

In 1993, Hudgins et al. [1] demonstrated an approach to control a multi-function prosthesis by using [MAV, MAVS, ZC, SSC, WL] feature set and ANN classifier. Zardoshti et al. [2] investigated [IAV, VAR, ZC, WAMP, v-order, AR model parameters and Histogram] feature set to control the upper extremity prostheses. They used nonparametric kNN classifier to test the quality of features spaces derived from EMG signal. Englehart et al. [3] showed that PCA is more powerful technique of feature reduction than Euclidean distance class separability- feature selection for timefrequency representations based feature sets.

Zecca et al. [4] presented the overview of different methods for controlling the artificial hands using EMG signal. Chan et al. [5] achieved classification accuracy using LDA classifier for RMS and AR coefficients feature sets. They compared two dimensionality reduction techniquesULDA and PCA. They obtained that ULDA outperforms PCA feature reduction. Al-Faiz et al. [6] used [IAV, MAV, MAV1, VAR, WL, WAMP] features with KNN algorithm to obtain the classification accuracy for virtual data created from EMG signal simulator. Fougner et al. [7] suggested an unambiguous taxonomy for the upper limb prosthesis control problem.

Tello et al. [8] used LDA and kNN classifier for myoelectric control of a prosthetic hand to rehabilitate amputee. Phinyomark et al. [9] performed feature extraction from $1^{\text {st }}$ difference of sEMG time series and concluded that the accuracy was higher as compare to features extracted from original signals. Omari et al. [10] extracted different features from a four channel-sEMG signal and analyzed them using LDA, quadratic discriminant analysis, and kNN. Kalwa et al. [11] used DWT based feature extraction scheme and kNN classifier to classify neuromuscular diseases. In [12], authors have evaluated a number of time- domain features. They achieved the most classification accuracy for the combination of [AR-order4, and WAMP] features and [MAV1, WL, AAC, ZC, and WAMP] features using LDA classifier. In present study authors have investigated the classification performance for the combination of features 
[MAV1, WL, AAC, ZC, and WAMP] by using MLPNN and kNN classifiers.

\section{MATERIALS AND METHODOLOGY}

In this study the eight- channel MES database acquired by Dr. Adrian D. C. Chan, Professor- Carleton University, Ottawa ON is used. MES database consists of sEMG signals for 7- limb motions: Hand-Close, Hand-Open, Pronation, Supination, Wrist-Flexion, Wrist-Extension, and Rest. These sEMG signals were amplified, filtered and finally sampled to produce discrete sEMG signals. Figure 1 shows the electrodes placement used in the eight-channel sEMG data acquisition. The details of the experimental setup and data acquisition are described in [5].

In present study the MES data is taken for ten subjects. For each subject 4- sessions were accomplished on separate days. In each session 6- trials were done. N01S1T1 shows the MES data of first subject for first session and first trial. In this way we used total 240 trials database (from N01S1T1 to N10S4T6) for this study.
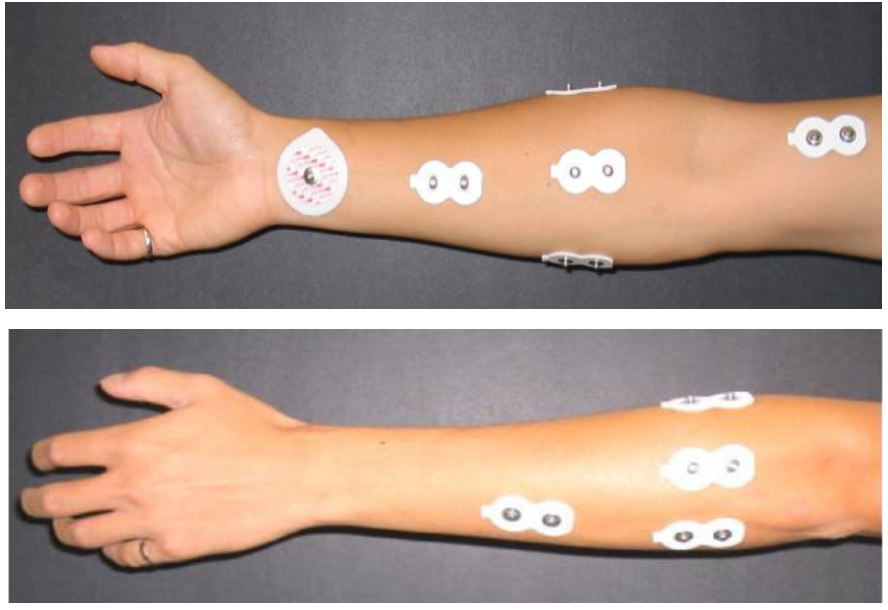

Fig. 1. The electrodes placement used in the eight-channel sEMG data acquisition [5].

The first phase in pattern recognition process is feature extraction; here input signal is converted into set of characteristic signal features. At this stage feature vector set [MAV1/WL/AAC/ZC/WAMP (threshold 0.01)] is obtained; thus we have total 40 feature vectors for eight channel data. Next min-max normalization is used which is defined as [normfeat $\left.\mathrm{t}_{\mathrm{i}}=\left(\mathrm{feat}_{\mathrm{i}}-\mathrm{min}_{\mathrm{i}}\right) /\left(\max _{\mathrm{i}}-\mathrm{min}_{\mathrm{i}}\right)\right]$; where feat $\mathrm{t}_{\mathrm{i}}$ is original feature value, normfeat $t_{i}$ is normalized feature values and $\max _{\mathrm{i}}$ and $\min _{\mathrm{i}}$ are the maximum and minimum values of every features in each channels.

The classification is next stage in pattern recognition; where the feature vectors are classified into seven classes. In present study the MLPNN and kNN classifiers are used. The commands are generated on the basis of decision made in this stage for controlling upper limb prosthesis.

\section{CLASSIFIERS}

\section{A. MLPNN Classifier}

ANN is a computational system motivated by the learning characteristics and structure of biological neural networks. MLPNN is the simplest and most widely used ANN technique. A logistic sigmoid function is used here as a nonlinear activation function. Both training and testing MES data are normalized by using min-max normalization; the training and test target vectors are converted into bipolar form; and then back propagation algorithm is used for training and testing. Then classification performance is obtained in following terms:

$$
\begin{aligned}
& \text { Sensitivity }=\frac{T P}{T P+F N} 100 \% \\
& \text { Specificity }=\frac{T N}{T N+F P} 100 \% \\
& \text { Accuracy }=\frac{T P+T N}{T P+T N+F P+F N} 100 \% \\
& \text { Positive predictivity }=\frac{T P}{T P+F P} 100 \%
\end{aligned}
$$

Four basic parameters true positive (TP), true negative $(\mathrm{TN})$, false negative (FN) and false positive (FP) are calculated from true class and predicted class data. Then the performance parameters mentioned above in equation 1 to 4 are obtained. Sensitivity is measures of the proportion of positives that are correctly identify positive observations. Specificity is a measure of the proportion of negatives that are truly negative. The accuracy of a test is the ratio of the number of correct assessments to the total number of assessments. The percent of all positive tests that are true positives shows positive predictivity.

\section{B. kNN Classifier}

$\mathrm{kNN}$ classifier is one of the simplest and the most important algorithm for EMG pattern recognition [2, 6, 8, 10, 11, and 15]. The algorithm consists of three stages: First the distances between a test sample and all training samples are calculated. Next pick out the k-nearest training samples to the test sample. Finally a class label is assigned by applying the majority rule to the $\mathrm{k}$ nearest samples [10]. In other words, $\mathrm{kNN}$ classifier is to classify unlabeled observations by assigning them to the class of the most similar labeled examples [13]. In $\mathrm{kNN}$ algorithm the nearest is defined in terms of a distance metric. Euclidean distance is used here. Euclidean distance between two points in n-dimensional space is defined by equation 5 .

$$
\operatorname{dist}\left(x_{1}, x_{2}\right)=\sqrt{\sum_{i=1}^{n}\left(x_{1 i}-x_{2 i}\right)^{2}}
$$

Where $x_{1}=\left(x_{11}, x_{12}, \ldots, x_{1 n}\right)$ and $x_{2}=\left(x_{21}, x_{22}, \ldots, x_{2 n}\right)$ are two records with $\mathrm{n}$ attributes. 


\section{RESUlTS AND DISCUSSION}

The classification performance is obtained for the feature vector set [MAV1/WL/AAC/ZC/WAMP (threshold 0.01)] using MLPNN and kNN classifiers. Table 1 shows the classification performance for ten subjects where the training data is the combination of N01S1T1/N02S1T1/N03S1T1/ N04S1T1/N05S1T1/N06S1T1/N07S1T1/N08S1T1/N09S1T1 /N10S1T1 datasets and the testing is done for all trials in each session (i.e. for all 240 trials). For eight- channels sEMG signal the dimensionality of feature vectors is 40 , so we have total 40 input neurons. Corresponding to seven limb motions we have 7 output neurons and the hidden neurons chosen are 10. For 1000 iterations (epochs) and different values of learning rate and momentum factor, the accuracy and mean square error is calculated. Using MLPNN classifier, an average classification accuracy of $(93.71 \pm 2.91) \%$ was achieved. The time taken by the CPU for training the network was found 6.94 minutes.

Table 1. CLASSIFICATION PERFORMANCE (\%) OBTAINED FOR MLPNN

\begin{tabular}{|c|c|c|c|c|c|c|c|}
\hline \multirow[b]{2}{*}{ 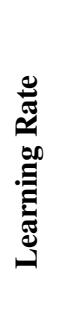 } & \multirow{2}{*}{ 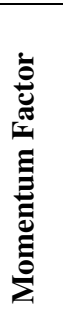 } & \multirow{2}{*}{ 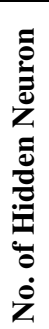 } & \multirow{2}{*}{ 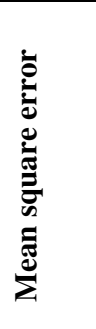 } & \multicolumn{4}{|c|}{$\begin{array}{c}\text { Performance Parameters } \\
(\text { Mean } \pm \text { SD })(\%)\end{array}$} \\
\hline & & & & 冚 & : & 莺 & 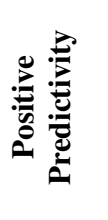 \\
\hline 0.48 & 0.11 & 10 & 0.4373 & $\begin{array}{r}93.71 \\
( \pm 2.91) \\
\end{array}$ & $\begin{array}{c}76.11 \\
( \pm 11.55)\end{array}$ & $\begin{array}{c}96.65 \\
( \pm 1.56)\end{array}$ & $\begin{array}{c}78.90 \\
( \pm 10.00)\end{array}$ \\
\hline 0.52 & 0.13 & 10 & 0.4352 & $\begin{array}{r}93.62 \\
( \pm 3.15)\end{array}$ & $\begin{array}{c}73.33 \\
( \pm 12.94)\end{array}$ & $\begin{array}{c}97.00 \\
( \pm 1.60)\end{array}$ & $\begin{array}{c}79.94 \\
( \pm 11.16)\end{array}$ \\
\hline 0.31 & 0.13 & 10 & 0.3549 & $\begin{array}{r}92.96 \\
( \pm 3.13)\end{array}$ & $\begin{array}{c}70.67 \\
( \pm 12.96)\end{array}$ & $\begin{array}{c}96.68 \\
( \pm 1.60)\end{array}$ & $\begin{array}{c}77.67 \\
( \pm 11.15)\end{array}$ \\
\hline
\end{tabular}

Next the classification accuracy is obtained using kNN for same feature set (Table 2). For classification, the first and fourth trials data are used for training purpose and the data from all trials is used for testing purpose. The window size used for feature extraction is $256 \mathrm{~ms}$. Both the training data and testing data have $50 \%$ overlap between windows.

Table 2. CLASSIFICATION ACCURACY (MEAN \pm SD $\%)$ OBTAINED FOR KNN

\begin{tabular}{|c|c|c|c|c|c|c|c|c|}
\hline $\mathbf{K}$ & 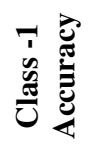 & 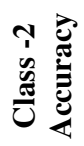 & 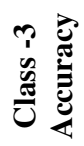 & 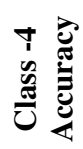 & 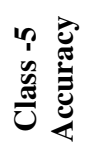 & 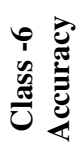 & 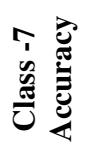 & 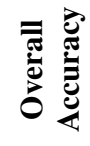 \\
\hline 3 & $\begin{array}{c}90.37 \\
(13.38)\end{array}$ & $\begin{array}{l}93.61 \\
(10.82)\end{array}$ & $\begin{array}{l}94.98 \\
(6.54)\end{array}$ & $\begin{array}{l}97.21 \\
(2.39)\end{array}$ & $\begin{array}{c}92.35 \\
(10.31)\end{array}$ & $\begin{array}{l}91.92 \\
(11.10)\end{array}$ & $\begin{array}{c}91.04 \\
(11.95)\end{array}$ & $\begin{array}{r}93.06 \\
(6.41)\end{array}$ \\
\hline 5 & $\begin{array}{c}90.32 \\
(12.85)\end{array}$ & $\begin{array}{c}93.59 \\
(10.39)\end{array}$ & $\begin{array}{l}94.51 \\
(6.51)\end{array}$ & $\begin{array}{l}96.86 \\
(2.20)\end{array}$ & $\begin{array}{c}92.42 \\
(10.05)\end{array}$ & $\begin{array}{c}92.19 \\
(10.48)\end{array}$ & $\begin{array}{c}91.12 \\
(11.64)\end{array}$ & $\begin{array}{r}92.99 \\
(5.99)\end{array}$ \\
\hline 7 & $\begin{array}{c}90.17 \\
(12.72)\end{array}$ & $\begin{array}{r}93.50 \\
(10.12)\end{array}$ & $\begin{array}{l}94.26 \\
(6.53)\end{array}$ & $\begin{array}{l}96.62 \\
(2.25)\end{array}$ & $\begin{array}{l}92.43 \\
(9.67)\end{array}$ & $\begin{array}{c}92.13 \\
(10.31)\end{array}$ & $\begin{array}{c}90.94 \\
(11.39)\end{array}$ & $\begin{array}{r}92.86 \\
(5.73) \\
\end{array}$ \\
\hline
\end{tabular}

The results showed that for feature set [MAV1/WL/AAC/ZC/WAMP] classification accuracy achieved is $(93.06 \pm 6.41) \%, \quad(92.99 \pm 5.99) \%, \quad$ and $(92.86 \pm 5.73) \%$ for $\mathrm{k}=3,5$, and 7 respectively using $\mathrm{kNN}$ classifier. The time taken by the CPU for classification was found around 0.16 seconds which is too low as compared to MLPNN. Figure 2 shows the confusion matrix for $\mathrm{k}=3,5$, and 7 respectively. A confusion matrix is used to summarize the performance of a classification algorithm.

Classification Accuracy [Training data: N01S1T1+N01S1T4/Testing data:N01S1T5/K=3] = 96.3663\%
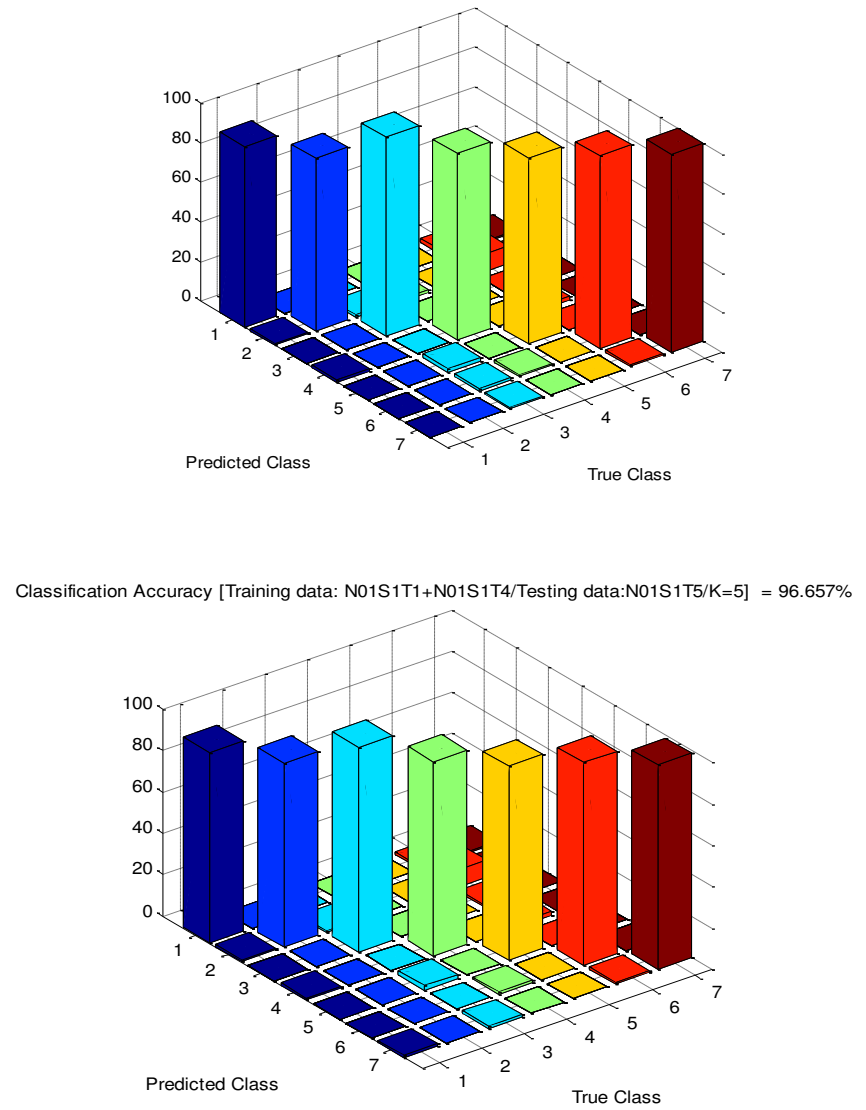

Classification Accuracy [Training data: N01S1T1+N01S1T4/Testing data:N01S1T5/K=7] $=97.3837 \%$

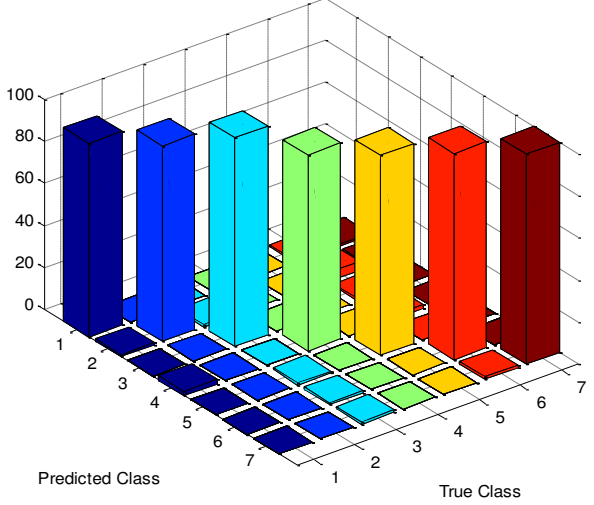

Fig. 2. Confusion Matrix for $\mathrm{k}=3, \mathrm{k}=5$, and $\mathrm{k}=7$ [training data N01S1T1+ N01S1T4/ testing data N01S4T5].

If we compare both the algorithms, both classifiers give an average accuracy of $93 \%$ but in terms of the time to train 
the system $\mathrm{kNN}$ give good performance with respect to MLPNN which is important in recognition systems; however one can achieve better accuracy with MLPNN by increasing the number of epochs for training.

\section{CONCLUSION}

Classification accuracy is obtained for myoelectric control of upper limb prosthesis using MLPNN and kNN classifiers. Using MLPNN classifier, an average classification accuracy of $(93.71 \pm 2.91) \%$ was achieved over 10 subjects and for kNN classifier, the average classification accuracy achieved was $(93.06 \pm 6.41) \%$. In terms of time and simplicity kNN is better than MLPNN.

\section{REFERENCES}

[1] Hudgins B, Parker P, Scott RN, "A new strategy for multifunction myoelectric control," IEEE Trans. Biomed. Eng., vol. 40, no.1, pp. 82-94, 1993.

[2] M. Zardoshti-Kermani, B. C. Wheeler, K.Badie, and R. M. Hashemi, "EMG feature evaluation for movement control of upper extremity prostheses," IEEE Trans. Rehabil. Eng., vol. 3, no. 4, pp. 324-333, Dec. 1995.

[3] Englehart, Kevin, et al. "Classification of the myoelectric signal using time-frequency based representations." Medical engineering \& physics 21.6 (1999): 431-438.

[4] Zecca, Micera, et al. "Control of multifunctional prosthetic hands by processing the electromyographic signal." Critical Reviews ${ }^{\mathrm{TM}}$ in Biomedical Engineering 30.4-6 (2002).

[5] Chan, Adrian DC, and Geoffrey C. Green. "Myoelectric control development toolbox." Proceedings of 30th conference of the Canadian medical \& biological engineering society. Vol. 1. 2007.
[6] Al-Faiz, Mohammed Z., Abduladhem A. Ali, and Abbas H. Miry. "A k-nearest neighbor based algorithm for human arm movements recognition using EMG signals." Energy, Power and Control (EPCIQ), 2010 1st International Conference on. IEEE, 2010.

[7] Fougner, Anders, et al. "Control of upper limb prostheses: terminology and proportional myoelectric control-a review." IEEE Transactions on neural systems and rehabilitation engineering 20.5 (2012): 663-677.

[8] Tello, Richard MG, et al. "Towards sEMG classification based on Bayesian and k-NN to control a prosthetic hand." Biosignals and Biorobotics Conference (BRC), 2013 ISSNIP. IEEE, 2013.

[9] Phinyomark, Angkoon, et al. "Feature extraction of the first difference of EMG time series for EMG pattern recognition." Computer methods and programs in biomedicine 117.2 (2014): 247-256.

[10] Al Omari, Firas, and L. Guohai. "Analysis of extracted forearm sEMG signal using LDA, QDA, K-NN classification algorithms'." The Open Automation and Control Systems Journal 6 (2014): 108116.

[11] Kalwa, Shravanti, and H. T. Patil. "Neuromuscular disease classification based on discrete wavelet transform of dominant motor unit action potential of EMG signal." Information Processing (ICIP), 2015 International Conference on. IEEE, 2015.

[12] Sachin Negi, Yatindra Kumar, and V. M. Mishra. "Feature extraction and classification for EMG signals using linear discriminant analysis." Advances in Computing, Communication, \& Automation (ICACCA) (Fall), International Conference on. IEEE, 2016.

[13] Zhang, Zhongheng. "Introduction to machine learning: k-nearest neighbors." Annals of Translational Medicine (2016).

[14] S. N. Sivanandam, S. Sumathi and S. N. Deepa, "Introduction to Neural Networks using MATLAB 6.0,"Tata McGraw-Hill Education (India) Pvt. Ltd.

[15] Al-Faiz, Mohammed Z., and Abbas H. Miry. Artificial Human Arm Driven by EMG Signal. INTECH Open Access Publisher, 2012.

[16] G. R. Naik Ed., Applications, Challenges, and Advancements in Electromyography Signal Processing, IGIGLOBAL publishers, USA, ISBN13:9781466660908,May-2014. 